\title{
Immunoregulation of a viral model of multiple sclerosis using the synthetic cannabinoid R(+)WIN55,212
}

\author{
J. Ludovic Croxford and Stephen D. Miller \\ Department of Microbiology-Immunology and the Interdepartmental Immunobiology Center, \\ Northwestern University Medical School, Chicago, Illinois, USA
}

\begin{abstract}
Theiler murine encephalomyelitis virus-induced demyelinating disease (TMEV-IDD) is a mouse model of chronic-progressive multiple sclerosis (MS) characterized by Th1-mediated CNS demyelination and spastic hindlimb paralysis. Existing MS therapies reduce relapse rates in 30\% of relapsing-remitting MS patients, but are ineffective in chronic-progressive disease, and their effects on disability progression are unclear. Experimental studies demonstrate cannabinoids are useful for symptomatic treatment of spasticity and tremor in chronic-relapsing experimental autoimmune encephalomyelitis. Cannabinoids, however, have reported immunosuppressive properties. We show that the cannabinoid receptor agonist, $\mathrm{R}(+)$ WIN55,212, ameliorates progression of clinical disease symptoms in mice with preexisting TMEV-IDD. Amelioration of clinical disease is associated with downregulation of both virus and myelin epitope-specific Th1 effector functions (delayed-type hypersensitivity and IFN- $\gamma$ production) and the inhibition of CNS mRNA expression coding for the proinflammatory cytokines, TNF- $\alpha$, IL1- $\beta$, and IL-6. Clinical trials investigating the therapeutic potential of cannabinoids for the symptomatic treatment of MS are ongoing, and this study demonstrates that they may also have potent immunoregulatory properties.
\end{abstract}

J. Clin. Invest. 111:1231-1240 (2003). doi:10.1172/JCI200317652.

\section{Introduction}

Multiple sclerosis (MS) is the most common human primary demyelinating disease of the CNS and is thought to be an autoimmune disease mediated by myelin-specific $\mathrm{CD} 4^{+}$Th1 cells (1). Although current disease-modifying therapeutic agents, IFN- $\beta$ and copaxone, reduce relapses in a portion of relapsingremitting MS patients, they are not curative, and their effectiveness in reducing disability progression in both relapsing-remitting and primary-progressive MS patients is unclear (2). Therefore, there is a need for novel therapeutic agents in the treatment of MS. The endocannabinoid network is a naturally occurring system, wherein endogenous ligands, such as anandamide (AEA), signal through two known cannabinoid receptors, $\mathrm{CB} 1$ and $\mathrm{CB} 2(3,4)$. Both of the cannabinoid receptors are present on immune cells, including $B$ lymphocytes, $T$ lymphocytes, macrophages, and natural killer cells, suggesting they may play a role in regulation

Received for publication December 17, 2002, and accepted in revised form February 18, 2003.

Address correspondence to: Stephen D. Miller, Department of Microbiology-Immunology, Northwestern University Medical School, 303 E. Chicago Avenue, Chicago, Illinois 60611, USA. Phone: (312) 503-1449; Fax: (312) 503-1154;

E-mail: s-d-miller@northwestern.edu.

Conflict of interest: The authors have declared that no conflict of interest exists.

Nonstandard abbreviations used: multiple sclerosis (MS); anandamide (AEA); $\Delta^{9}$-tetrahydrocannabinol $\left(\Delta^{9}\right.$-THC); experimental allergic encephalomyelitis (EAE); Theiler murine encephalomyelitis virus (TMEV); TMEV-induced demyelinating disease (TMEV-IDD); proteolipid protein (PLP); delayed-type hypersensitivity (DTH). of immune functions (5). Numerous in vitro studies have described the immunomodulatory properties of cannabinoids, particularly $\Delta^{9}$-tetrahydrocannabinol $\left(\Delta^{9}\right.$-THC), the major psychoactive constituent of marijuana (6). Clinical trials to determine the symptomatic therapeutic potential of $\Delta^{9}$-THC on spasticity, bladder dysfunction, and mobility in MS patients are underway (7). Cannabinoid receptor agonists have also been shown to alleviate both tremor and spasticity, associated with neurological dysfunction in experimental allergic encephalomyelitis (EAE), an animal model of MS $(8,9)$. No studies to date, however, have determined the therapeutic immunosuppressive properties of cannabinoids in viral autoimmune disease models.

MS is thought to have a infectious etiology based on epidemiological evidence that viral infections may play a role in the initiation and/or exacerbation of clinical disease $(10,11)$. Theiler murine encephalomyelitis virus (TMEV) is a single-stranded RNA virus belonging to the Picornaviridae family (12). TMEVinduced demyelinating disease (TMEV-IDD) is a natural chronic-progressive CNS demyelinating disease of susceptible strains of mice, with similarities to primary-progressive MS $(13,14)$. Although TMEV-IDD is initiated by viral capsid protein-specific $\mathrm{CD}^{+} \mathrm{T}$ cells targeting virus persisting in CNS microglia, autoreactive myelin-specific $\mathrm{CD}^{+} \mathrm{Th} 1$ responses arise by epitope spreading and contribute to chronic pathology $(15,16)$. Myelin destruction is mediated largely by the production of the proinflammatory cytokines, IFN- $\gamma$ and TNF- $\alpha$, secreted from autoreactive $\mathrm{T}$ cells and macrophages. Blockade of these inflammatory mediators with TNF-receptor fusion 
proteins or immunomodulation using anti-inflammatory agents such as IL-10 are effective therapies in EAE (17-19).

We investigated the immunosuppressive potential of $\mathrm{R}(+)$ WIN55,212, a cannabinoid receptor agonist, as a treatment for the clinical autoimmune disease associated with TMEV infection. The results show that $\mathrm{R}(+)$ WIN55,212 is an effective therapeutic agent for ongoing TMEV-IDD and that R(+)WIN55,212 treatment inhibits the differentiation of Th 1 effector cells. The data suggest that the potent immunosuppressive properties of $\mathrm{R}(+)$ WIN55,212 may have significant therapeutic potential in ameliorating disability progression in MS patients currently undergoing clinical trials with cannabinoids for the treatment of spasticity and tremor.

\section{Methods}

Infection of SJL mice with TMEV. Five- to six-week-old female SJL mice were purchased from Harlan Laboratories (Bethesda, Maryland, USA) and housed in the Northwestern University Center for Comparative Medicine (Chicago, Illinois, USA). Mice were infected by intracerebral injection of $3 \times 10^{7} \mathrm{PFU}$ of wildtype TMEV (BeAn 8386 strain) and scored at daily intervals on a clinical scale of $0-5: 0$, no signs of disease; 1 , mild gait abnormalities; 2 , severe gait abnormalities; 3 , paralysis in one limb; 4 , more than one paralyzed limb; 5, moribund.

Peptides. Proteolipid protein (PLP) peptide PLP $_{139-151}$ (HSLGKWLGHPDKF) and the TMEV peptide VP2 $2_{70-86}$ (WTTSQEAFSHIRIPLPH) were purchased from Peptides International Inc. (Louisville, Kentucky, USA). The amino acid composition was verified by mass spectrometry, and purity was assessed by HPLC.

Administration of WIN55,212. R(+)WIN55,212 and S(-)WIN55,212 (Sigma-Aldrich, St. Louis, Missouri, USA) were dissolved in 10\% Tween-80 (Sigma-Aldrich) and PBS to a final concentration of $4 \mathrm{mg} / \mathrm{ml}$ or $1 \mathrm{mg} / \mathrm{ml}$. The mixture was then vortexed and passed through a 2 -gauge needle. Similar preparations without active compounds were used as vehicle controls. Mice were treated with suspensions $(0.1 \mathrm{ml})$ injected intraperitoneally. Groups of TMEV-infected mice $(n=5-10)$ were treated with either a high $(20 \mathrm{mg} / \mathrm{kg})$ or low $(5 \mathrm{mg} / \mathrm{kg})$ dose of WIN55,212 at varying stages of disease.

Delayed-type bypersensitivity response. Delayed-type hypersensitivity (DTH) responses were elicited by injecting mice subcutaneously with $5 \mu \mathrm{g}$ of the challenge peptides, $\mathrm{PLP}_{139-151}$ or VP2 $70-86$, into alternate ears following measurement of ear thickness using a Mitutoyo model 7326 engineer's micrometer (Schlesinger's Tools, Brooklyn, New York, USA). Twenty-four hours after peptide challenge, the ears were remeasured, and differences in ear swelling over pre-challenge thickness were expressed in units of $10^{-4}$ inches plus or minus SEM.

$T$ cell proliferation and cytokine analysis. Spleens were removed from infected mice $(n=2)$ at various times following infection. $\mathrm{T}$ cell proliferation and cytokine analysis were performed as described previously (20). Proliferation was determined from triplicate wells for each peptide concentration and then expressed as $\Delta$ counts per minute. For IFN- $\gamma$ and IL- 4 cytokine analysis, a duplicate set of proliferation wells were used to collect supernatants at 48 and $72 \mathrm{~h}$, and cytokine concentrations were determined by ELISA.

Virus plaque assays. Brains were removed from infected mice either on day 7 following day 0-5 R(+)WIN55,212 treatment or on day 33 after infection following day 26-31 R(+)WIN55,212 treatment. Organs were weighed, homogenized, and diluted in serum-free DMEM media. Virus plaque assays were performed as described previously (20). Plaques were counted on each plate and multiplied by the dilution and corrected for the quantity of tissue used to give a value in plaque forming units per milligram of tissue.

$R T-P C R$. Following the induction of TMEV-IDD, mice $(n=2$ per time point) were anesthetized and perfused with $50 \mathrm{ml}$ of PBS. Spinal cords were extruded by flushing the vertebral canal with PBS. Isolation of mRNA from tissue was performed as described previously (21). First-strand cDNA was generated from $2 \mu \mathrm{g}$ total RNA using Advantage-RT Kit (CLONTECH Laboratories Inc., Palo Alto, California, USA) using 20 pmol oligo(dT) primer, per the manufacturer's provided protocol, in a total volume of $20 \mu \mathrm{l}$. Cytokine PCR and densitometry was performed as described previously (21). The sum intensity and band area were determined for each competitor/wild-type amplicon pair, and a ratio of wild-type/competitor intensity was determined for all cytokines and adjusted to each sample's housekeeping gene, HPRT. The relative levels of mRNA present were measured as the percentage of intensity of the HPRT control for that sample.

FACS analysis of splenic leucocytes. Splenocyte populations were stained using anti-CD4, anti-CD8, or anti-B220-APC-conjugated Ab, anti-F4/80-FITC-conjugated $\mathrm{Ab}$, or anti-CD4-PerCP-conjugated $\mathrm{Ab}$ and anti-CD25-APC-conjugated Ab (PharMingen, San Diego, California, USA). Cells were labeled, washed, resuspended in PBS containing 1\% FCS and analyzed by flow cytometry on a FACStar (Becton Dickinson Immunocytometry Systems, Mountain View, California, USA). The total number of cells of each population was analyzed individually from three mice per group and then plotted as the mean total number of cells plus or minus SEM.

Statistics. Clinical severity results were presented as the mean group clinical score and the statistical difference calculated by the Mann-Whitney nonparametric ranking test. Analysis of DTH responses, virus titers, proliferation, IFN- $\gamma$ ELISA, and FACS analysis was performed using the two-tailed Student $t$ test.

\section{Results}

Cannabinoid therapy delays the onset and reduces clinical severity of TMEV-IDD. The intraperitoneal administration of R(+)WIN55,212 $(20 \mathrm{mg} / \mathrm{kg})$ at the time of 
a

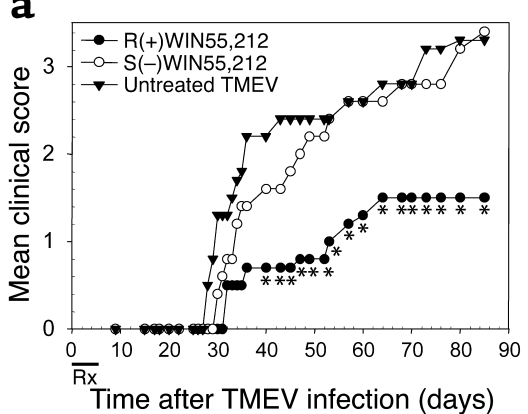

b

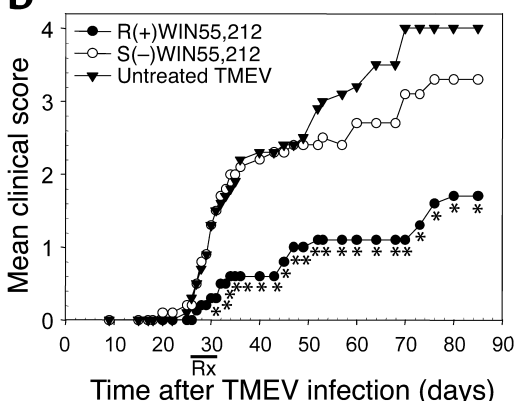

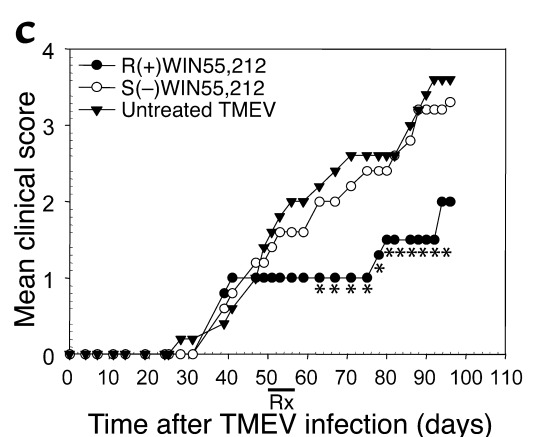

Figure 1

Cannabinoid receptor agonism ameliorates the clinical severity of established TMEV-induced demyelinating disease. Treatment with

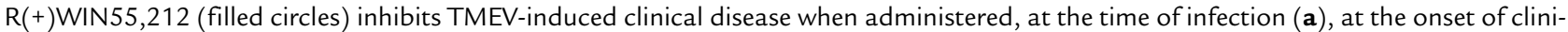
cal disease (b), or during established disease $(\mathbf{c})$. Rx, duration of administration of WIN55,212 to mice. ${ }^{*}$ Significant inhibition $(P<0.05)$ of clinical disease score in R(+)WIN55,212-treated mice compared with S(-)WIN55,212 (open circles) or untreated TMEV mice (filled triangles). Data are representative of three separate experiments.

TMEV infection (day 0-5) significantly delayed the onset of clinical disease and ameliorated both the incidence and severity of clinical disease at equivalent time points up to day 85 , compared with either mice treated with the inactive enantiomer S(-)WIN55,212 or untreated TMEV-infected mice $(P<0.05)$ (Figure 1a). Treatment with $\mathrm{R}(+)$ WIN55,212 between postinfection day 26-31, i.e., at the onset of TMEV-induced clinical disease, also ameliorated the clinical severity of TMEV-IDD $(P<0.05)$ (Figure $1 \mathrm{~b})$. In addition, $\mathrm{R}(+)$ WIN55,212 treatment during established disease (postinfection days 50-55) stabilized clinical severity at grade 1 for over 20 days compared with the continued increase of clinical severity in control mice (Figure 1c). Importantly, there was no significant difference in severity, incidence, or onset of TMEVinduced disease between untreated TMEV-infected mice and those treated with the S(-)WIN55,212 enantiomer (Figure 1) or vehicle-treated mice (data not shown). Treatment of TMEV-infected mice with a lower dose of R(+)WIN55,212 (5 or $1 \mathrm{mg} / \mathrm{kg}$ ) at the time of TMEV infection (postinfection day 0-5) or at the onset of clinical disease (postinfection day 26-31) was unsuccessful at reducing either the incidence or severity of disease compared with control mice (data not shown), indicating the dose dependency of the protection. In addition, mice treated on alternative days (between postinfection day $0-8$ or 26-34) also did not show any significant reduction in disease severity (data not shown).

$R(+)$ WIN55,212 treatment increases the viral load in brain tissue at early, but not late time points. To determine whether R(+)WIN55,212 treatment increased the susceptibility of SJL/J mice to TMEV infection, TMEV viral titers were measured in the brains of mice 2 days after the last treatment dose at each of the time points studied (postinfection day 0-5 and 26-31). The viral load was found to be significantly increased in mice treated with $\mathrm{R}(+)$ WIN55,212 at the early time point $(P<0.05)$ (Figure 2a), however viral titers following $\mathrm{R}(+)$ WIN55,212 treatment initiated at the onset of clinical disease were similar to controls (Figure $2 b$ ). a

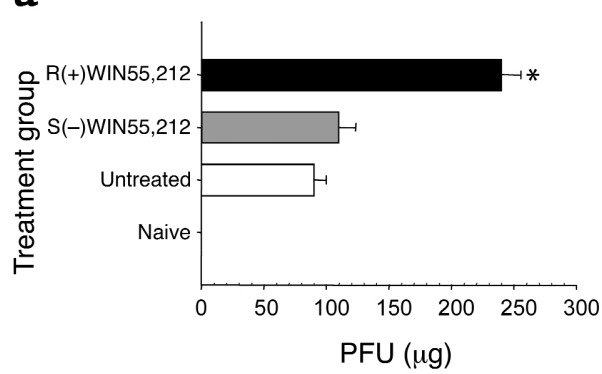

b

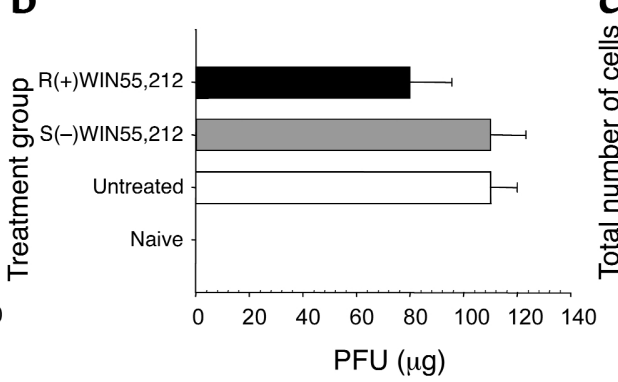

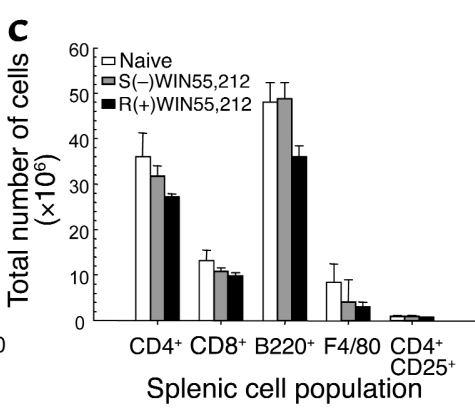

Figure 2

Cannabinoid treatment increases the susceptibility of mice to TMEV infection and is not cytotoxic to splenocytes. R(+)WIN55,212 treatment of mice at the time of infection (a) (black bars) showed a significant increase in CNS virus titers compared with either S(-)WIN55,212 (gray bars) or untreated (white bars) TMEV-infected mice. Virus titers were not different from controls in mice treated with R(+)WIN55,212 at the onset of disease (b). *Significant increase in viral load in R(+)WIN55,212-treated mice compared with either S(-)WIN55,212 or untreated TMEV mice $(P<0.05)$. No PFUs were observed in naive mice. R(+)WIN55,212 treatment has no cytotoxic effect, as measured by FACS analysis of splenic $\mathrm{CD}^{+}, \mathrm{CD}^{+}, \mathrm{B} 220^{+}$, and $\mathrm{CD} 4^{+} \mathrm{CD} 25^{+}$lymphocytes, and $\mathrm{F} 4 / 80^{+}$macrophage populations compared with $\mathrm{S}(-) \mathrm{WIN} 55,212$ or naive mice $(\mathbf{c})$. Results are expressed as the mean total number of cells $(n=3$ per group $) \pm$ SEM. 

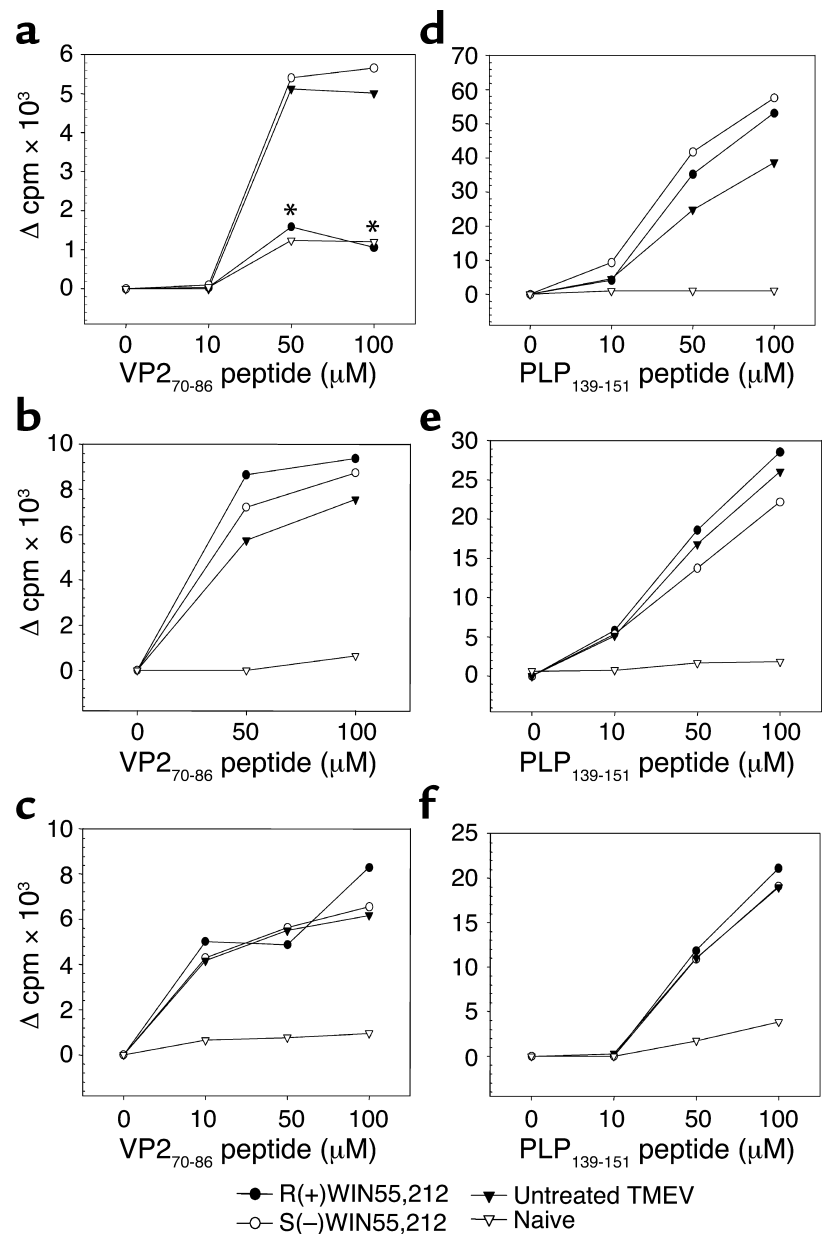

In vivo administration of $R(+)$ WIN55,212 is not toxic to lymphocytes. To determine if the disease ameliorating effect of R(+)WIN55,212 was due to a cytotoxic effect on lymphocytes, we examined the effects of the treatment on splenic lymphocyte subpopulations by FACS analysis (Figure $2 \mathrm{c}$ ). Groups of naive mice $(n=3)$ mice were treated with either $20 \mathrm{mg} / \mathrm{kg}$ of R(+)WIN55,212

\section{Figure 3}

Effects of cannabinoid receptor agonism on $\mathrm{T}$ cell proliferation depends upon the cellular activation state. Early R(+)WIN55,212 treatment (filled circles) (postinfection day $0-5$ ) significantly inhibited the proliferation of $\mathrm{T}$ lymphocytes compared with $\mathrm{S}(-)$ WIN55,212 (open circles) and untreated TMEV-infected mice (filled triangles) upon rechallenge with the TMEV VP2 ${ }_{70-86}$ peptide (postinfection day 8) (a), but not $\mathrm{PLP}_{139-151}$ (d). When measured at postinfection day $40, \mathrm{R}(+) \mathrm{WIN} 55,212$ treatment did not inhibit $\mathrm{T}$ lymphocyte proliferation to either $\mathrm{VP} 2_{70-86}$ or $\mathrm{PLP}_{139-151}$ peptides during established disease (postinfection day 26-31 or 50-55) (b, c, e, and f). ${ }^{*} P<0.05$, compared with either $\mathrm{S}(-)$ WIN55,212 or untreated mice.

or S(-)WIN55,212 intraperitoneally for 5 days. Twenty-four hours after the last treatment, the percentages and numbers of $\mathrm{CD}^{+} \mathrm{T}$ cells, $\mathrm{CD} 8^{+} \mathrm{T}$ cells, $\mathrm{B}$ cells, and $\mathrm{F} 4 / 80^{+}$macrophages were determined by FACS analysis. There was no significant changes in the total number of cells per spleen or the number of cells within each subpopulation (Figure 2c).

$\mathrm{R}(+)$ WIN55,212 treatment inhibits the proliferative capacity of naive, but not activated $\mathrm{T}$ cells. Mice treated with $\mathrm{R}(+)$ WIN55,212 between postinfection day $0-5$ demonstrated a significantly reduced $\mathrm{T}$ cell proliferative response following rechallenge with the immunodominant TMEV epitope (VP2 ${ }_{70-86}$ ) compared with controls $(P<0.05)$ (Figure 3a). There was no significant effect on $\mathrm{VP} 2_{70-86}$-specific proliferative responses, however, in mice with established clinical disease (postinfection day 32 or 56) treated with $\mathrm{R}(+)$ WIN55,212 (Figure 3, $\mathrm{b}$ and c). Following TMEV infection, peripheral autoreactive $\mathrm{PLP}_{139-151}$-specific responses can be detected beginning at postinfection day 40-50. Although early R(+)WIN55,212 treatment (postinfection day 0-5) inhibited VP2 $2_{70-86}$ proliferative responses, there was no effect on the proliferative responses to $\mathrm{PLP}_{139-151}$ when measured at postinfection day 40 (Figure $3 \mathrm{~d}$ ). In fact, $\mathrm{R}(+)$ WIN55,212 treatment at all three time points was unsuccessful at
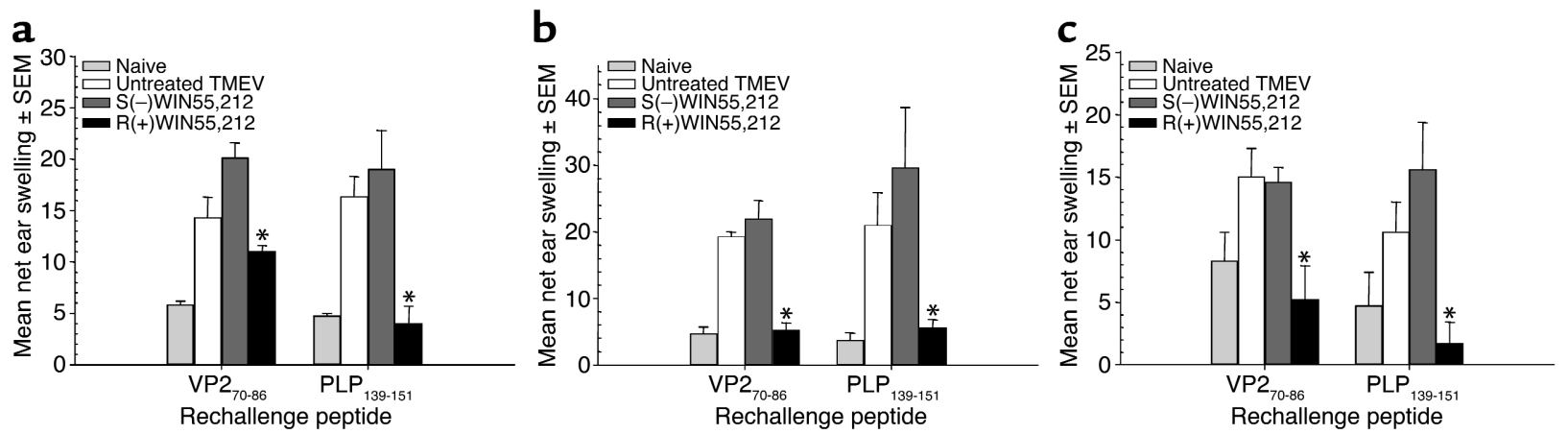

Figure 4

Cannabinoid treatment inhibits both virus and myelin autoimmune inflammatory DTH responses. Treatment of mice with $\mathrm{R}(+)$ WIN55,212 (black bars) at the time of TMEV infection (a), at the onset of clinical disease (b), or during established disease (c), significantly reduces DTH responses to rechallenge with either the immunodominant TMEV peptide VP2 $70-86$ or the autoreactive myelin $\mathrm{PLP}_{139-151}$ peptide. * Significant reduced DTH responses compared with either S(-)WIN55,212 (dark gray bars) or untreated TMEV-infected (white bars) mice $(P<0.05)$. Naive mice (light gray bars) show a low background response to either peptide. 

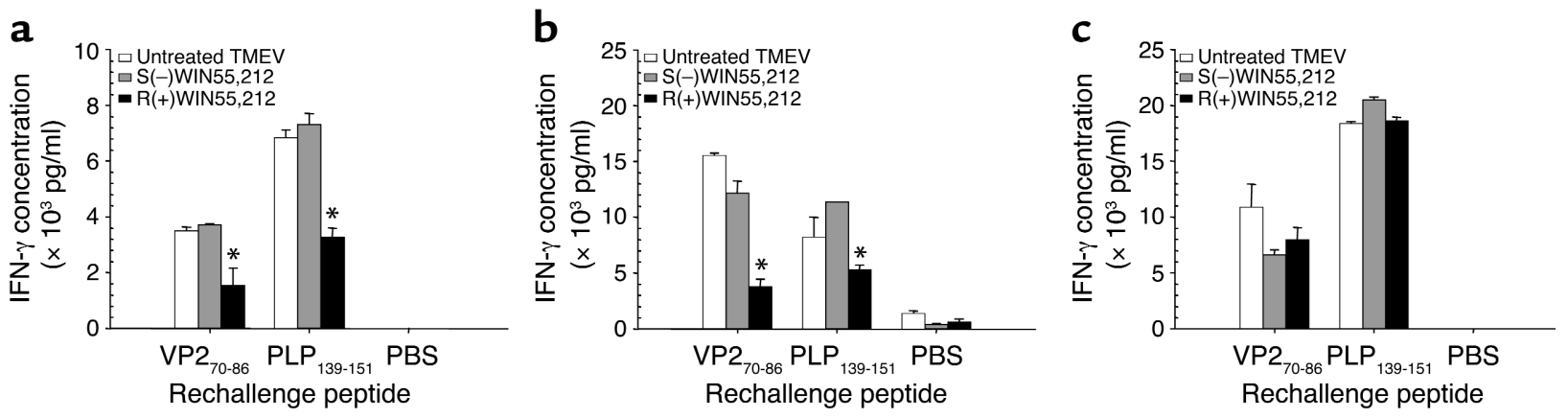

Figure 5

Cannabinoid receptor agonism inhibits Th1-mediated IFN- $\gamma$ cell secretion. Treatment with R(+)WIN55,212 at the time of infection (a) or at the onset of clinical disease (b) significantly inhibited IFN- $\gamma$ secretion from T lymphocytes rechallenged with either VP2 $70-86$ or PLP $139-151$ compared with either S(-)WIN55,212 (gray bars) or untreated TMEV-infected mice (white bars). R(+)WIN55,212 treatment during established disease (c) had no significant effect on IFN- $\gamma$ secretion. ${ }^{*}$ Cytokine response was significantly inhibited compared with either $\mathrm{S}(-)$ WIN55,212 or untreated TMEV-infected mice. $P<0.05$.

inhibiting the proliferative capacity of activated

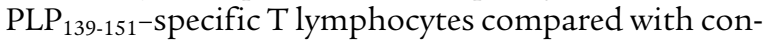
trols (Figure 3, d-f).

$D T H$ responses are transiently inbibited by $R(+)$ WIN55,212 treatment. DTH measures the in vivo release of Th1-type cytokines such as IFN- $\gamma$, and other mediators of tissue injury and inflammation, from recruited inflammatory Th1 cells. Following treatment with R(+)WIN55,212 or appropriate controls, mice were rechallenged with either the immunodominant TMEV VP2 ${ }_{70-86}$ epitope or the myelin PLP $_{139-151}$ epitope (Figure 4). DTH recall responses were measured at postinfection day 38 for the day $0-5$ treatment groups and 3 days after $\mathrm{R}(+)$ WIN55,212 treatment at the other time points. The DTH responses to VP2 $2_{70-86}$ and PLP ${ }_{139-151}$ were significantly reduced compared with controls $(P<0.05)$ (Figure $4, \mathrm{a}-\mathrm{c})$ at all time points. In most cases, the $\mathrm{R}(+)$ WIN55,212-treated mice exhibited a total lack of DTH responses. In addition, S(-)WIN55,212-treated and untreated TMEV-infected mice exhibited similar DTH responses at all time points suggesting no inhibitory effect of either the $\mathrm{S}(-)$ enantiomer of WIN55,212 or the vehicle (Figure 4, a-c).
$R(+)$ WIN55,212 treatment inhibits Th1-type cytokine secretion from both virus-specific and autoreactive $P L P_{139-151-\text { spe- }}$ cific $T$ cells. The ability of $\mathrm{R}(+)$ WIN55,212-treated mice to mount Th1-type cytokine responses was measured in vitro by IFN- $\gamma$ ELISA. Splenocyte cultures from TMEV-infected mice were rechallenged with either VP2 $2_{70-86}$ or $\mathrm{PLP}_{139-151}$. R(+)WIN55,212 treatment of mice either at the time of infection (postinfection day $0-5$ ) or at the onset of clinical disease (postinfection day 26-31), significantly reduced the capacity of $T$ lymphocytes to secrete IFN- $\gamma$ in response to either $\mathrm{PLP}_{139-151}$ or VP2 $2_{70-86}(P<0.05)$ (Figure 5 , a and $b$ ). $\mathrm{R}(+)$ WIN55,212 treatment during established disease, however, did not significantly inhibit IFN- $\gamma$ secretion of peripheral $\mathrm{T}$ cells in response to either $\mathrm{PLP}_{139-151}$ or VP2 ${ }_{70-86}$ (Figure 5c). In all experiments S(-)WIN55,212 had little effect upon IFN- $\gamma$ secretion compared with untreated TMEV-infected mice (Figure 5, a-c).

$R(+)$ WIN55,212 treatment results in reduction of $m R N A$ expression for proinflammatory and antiviral mediators in the spinal cord. Following $\mathrm{R}(+) \mathrm{WIN} 55,212$ treatment at the onset of infection (postinfection day 0-5), spinal cords a

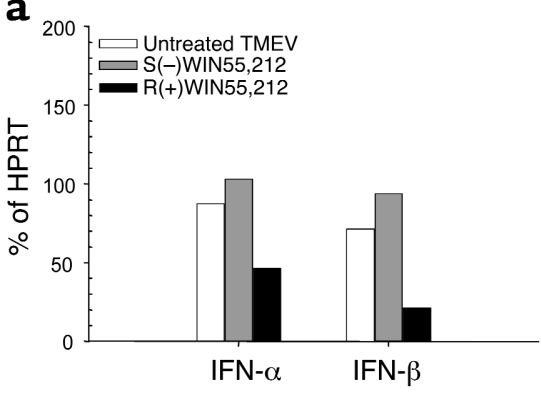

b

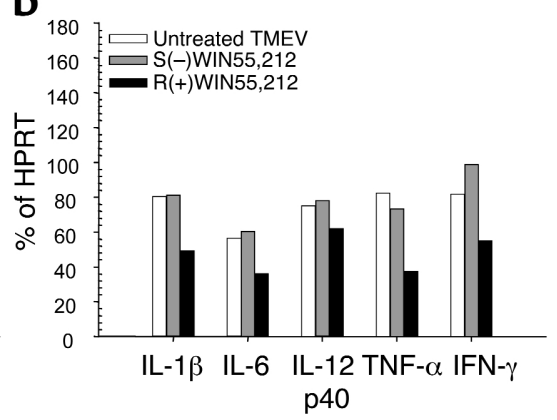

c

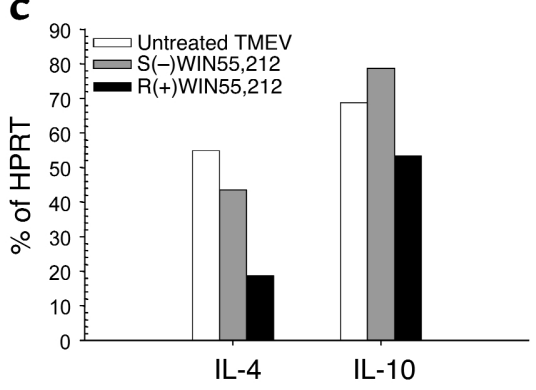

Figure 6

Cannabinoid treatment inhibits mRNA coding for both antiviral and Th1- and Th2-type inflammatory mediators in the CNS. R(+)WIN55,212 treatment at the time of infection (day 0-5) inhibited spinal cord mRNA coding for the innate antiviral mediators, IFN- $\alpha$, and IFN- $\beta$ (a). $\mathrm{R}(+)$ WIN55,212 treatment at the onset of disease inhibited spinal cord mRNA coding for the proinflammatory Th1 mediators, IL-1 $\beta$, IL-6, TNF- $\alpha$, and IFN- $\gamma$, but not the IL-12 p40 subunit (b). Treatment at disease onset also inhibited mRNA coding for the Th2 cytokine IL-4, but not IL-10 (c). The mRNA levels are presented as the percentage of intensity of the HPRT control for that sample. 
Table 1

Differential inhibition of immune response parameters following $R(+)$ WIN55,212 treatment ofTMEV-IDD is dependent on the stage of disease

\begin{tabular}{|c|c|c|c|c|c|c|c|}
\hline \multirow[b]{2}{*}{ Stage of disease } & \multirow[b]{2}{*}{ Inhibition of clinical disease } & \multicolumn{2}{|c|}{ Inhibition of DTH } & \multicolumn{2}{|c|}{ Inhibition of T cell proliferation } & \multicolumn{2}{|c|}{ Inhibition of IFN- $\gamma$ secretion } \\
\hline & & VP2 ${ }_{70-86}$ & $\mathrm{PLP}_{139-151}$ & VP2 $70-86$ & $\mathrm{PLP}_{139-151}$ & VP2 ${ }_{70-86}$ & $\mathrm{PLP}_{139-151}$ \\
\hline $\begin{array}{l}\text { Initiation } \\
\text { (postinfection day 0-5) }\end{array}$ & + & + & + & $++^{A}$ & $-{ }^{A}$ & + & + \\
\hline $\begin{array}{l}\text { Onset } \\
\text { (postinfection day 26-31) }\end{array}$ & + & + & + & - & - & + & + \\
\hline $\begin{array}{l}\text { Late } \\
\text { (postinfection day 50-55) }\end{array}$ & + & + & + & - & - & - & - \\
\hline
\end{tabular}

AVP2 $70-86$ proliferation determined at postinfection day 6 and PLP ${ }_{139-151}$ proliferation at postinfection day 36.

were removed on postinfection day 7 and analyzed by RT-PCR for the presence of cytokine mRNA. $\mathrm{R}(+)$ WIN55,212 treatment led to a significant reduction in the spinal cord expression of mRNA for the innate antiviral mediators, IFN- $\alpha$ and IFN- $\beta$, compared with either control mice, as measured by semiquantitative RT-PCR (Figure 6a). R(+)WIN55,212 treatment at the onset of clinical disease (postinfection day 26-31) resulted in a significant reduction in spinal cord mRNA expression of the proinflammatory mediators, IL-1 $\beta$, IL-6, TNF- $\alpha$, IFN- $\gamma$ (Figure 6b), and IL-4 (Figure 6c) compared with controls. Interestingly, $\mathrm{R}(+)$ WIN55,212 treatment had no effect on mRNA levels in the CNS coding for the IL-12 p40 subunit (Figure $6 \mathrm{~b}$ ) or for IL-10 (Figure 6c).

\section{Discussion}

Based on the numerous reports of the in vitro immunomodulatory effects of cannabinoids, we investigated the immunotherapeutic effects of $\mathrm{R}(+)$ WIN55,212 in TMEV-IDD, a chronic-progressive autoimmune model of MS. SJL mice were treated with $\mathrm{R}(+)$ WIN55,212 at three different time points: (a) concomitant with TMEV infection to study effects on virus infection/persistence of the CNS and the associated innate (type 1 IFN) and adaptive $\left(\mathrm{CD} 4^{+} \mathrm{T}\right.$ cell) antiviral immune responses; (b) at the onset of clinical disease to determine the immunosuppressive effect upon antiviral and autoreactive anti-myelin $\mathrm{T}$ cell responses; (c) during established disease, which has clinical relevance for the treatment of MS. We found that intraperitoneal treatment with $\mathrm{R}(+)$ WIN55,212 at all times significantly inhibited progression of clinical disease. The short course of $\mathrm{R}(+)$ WIN55,212 treatment ( 6 consecutive days) appeared to be effective for at least 30 days after treatment. After 30 days, treated mice began to show signs of increasing clinical severity, although still significantly less severe than controls. Interestingly, administration of R(+)WIN55,212 every other day (6 total injections) had little effect on the disease course of TMEV-IDD (data not shown), suggesting $\mathrm{R}(+)$ WIN55,212 has a short active half-life in vivo. Importantly, R(+)WIN55,212 is not toxic to lymphocytes as the total number of $\mathrm{CD} 4^{+}, \mathrm{CD} 8^{+}, \mathrm{CD}^{+} \mathrm{CD} 25^{+}$, and $\mathrm{B} 220^{+}$lymphocytes, and $\mathrm{F} 4 / 80^{+}$macrophages in the spleen, were unchanged following treatment. In addition, there was no indication of toxicity in treated versus control groups as measured by a lactose dehydrogenase cytotoxicity assay (data not shown). In contrast to existing treatments for MS, side-effects of $\mathrm{R}(+)$ WIN55,212 treatment were mild, consisting of psychoactive effects, primarily ataxia, between 1 and 30 minutes after administration of $20 \mathrm{mg} / \mathrm{kg}$. Similar adverse events were reported in a double-blind study on the safety and efficacy of oral $\Delta^{9}$-THC for the symptomatic treatment of MS (22).

The therapeutic effect of R(+)WIN55,212 was associated with a reduced capacity of $\mathrm{CD}^{+} \mathrm{T}$ cells to differentiate to Th1 effector cells. The Th1 cytokines IFN- $\gamma$ and TNF- $\alpha$ promote the activation of the immune system. $\mathrm{R}(+)$ WIN55,212 treatment significantly reduced the ability of mice to mount DTH responses to either VP2 $2_{70-86}$ or $\mathrm{PLP}_{139-151}$ following treatment at all three time points. In addition, IFN- $\gamma$ secretion from $\mathrm{CD}^{+} \mathrm{T}$ lymphocytes from $\mathrm{R}(+)$ WIN55,212-treated mice was reduced compared with untreated or S(-)WIN55,212-treated mice. R(+)WIN55,212 treatment at the onset of clinical disease (postinfection day 26-31) also significantly inhibited levels of spinal cord mRNA coding for a number of inflammatory mediators (IL-1 $\beta$, IL-6, TNF- $\alpha$, and IFN- $\gamma$ ) important in the induction and progression of TMEV-IDD, EAE, and presumably MS (18, 23-26). Previous studies from our group have demonstrated that mRNA coding for the proinflammatory cytokines IFN- $\gamma$ and TNF- $\alpha$, are expressed at equivalent levels during the onset of disease (postinfection day 33) and late stage disease (postinfection day 103) (21).

$\mathrm{R}(+)$ WIN55,212 mediates its effects through CB1 and CB2 receptors. Therefore, some downregulatory effects of $\mathrm{R}(+)$ WIN 55,212 on CNS cytokine mRNA transcription levels may be directed toward resident CNS cells such as astrocytes, microglial, and endothelial cells. We are currently investigating the effects of $\mathrm{R}(+)$ WIN55,212 on cytokine secretion of TMEV-infected glial cells. In addition, recent studies have shown that human dendritic cells express both CB1 and CB2 receptors and fatty acid amide hydrolase, which hydrolyzes endocannabinoids (27). In addition, $\Delta^{9}$-THC can inhibit in vitro $T$ cell proliferation and Th1 
polarization in dendritic/T cell cocultures (28). This indirectly supports the findings in this study, whereby cannabinoids can downregulate the immune response by inhibiting Th1 polarization and therefore effector function during TMEV infection and autoimmune disease. We are currently investigating potential direct immunomodulatory effects of cannabinoids on dendritic cells during TMEV-IDD. Although the endogenous cannabinoid, AEA, can inhibit TMEV-induced TNF- $\alpha$ secretion from astrocytes, IL- 6 is upregulated $(29,30)$. Differences in IL-6 production may be due to the effects of AEA versus R(+)WIN55,212 or the differences between in vitro versus in vivo experiments. Surprisingly, $\mathrm{R}(+)$ WIN55,212 had no effect on the levels of mRNA coding for the IL-12 p40 subunit, which is involved in Th1 induction. Although IL-1 $\beta$, IL-6, IL-12 $\mathrm{p} 40$, and TNF- $\alpha$ are all regulated by NF- $\mathrm{\kappa B} /$ Rel transcription factors, the IL-12 p40 subunit may require c-Rel, a transcription factor not required for transcription of the other cytokines (31). This suggests that $\mathrm{R}(+)$ WIN55,212 may affect different transcription factors, which in turn modulate the expression of cytokines produced. Cannabinoid treatment may also induce the expression of Th2 cytokines, such as IL-4 and IL-10 (32), which have therapeutic effects in EAE $(17,19)$. Treatment with $\Delta^{9}$-THC for BALB/c mice infected with Legionella pneumophila suppressed IFN- $\gamma$ secretion and induced IL-4 expression (32). In this study we found no evidence of Th2 cytokine induction, either IL-4 or IL-10, by ELISA or ELISPOT from splenocyte cultures from R(+)WIN55,212-treated mice at all treatment times (data not shown). This may reflect the reduced capacity of SJL mice to produce IL-4 (33). In contrast, mRNA analysis of the spinal cord at the onset of clinical disease showed a decrease in IL-4, but not IL-10, mRNA. Since IL-4 is predominantly derived from Th2 cells, the effects of R(+)WIN55,212 are apparently directed predominantly toward the regulation of Th1 responses. IL-10 is expressed by a number of cell types apart from $\mathrm{T}$ lymphocytes and is expressed constitutively by resident CNS cells $(19,34)$, which may account for its relatively unchanged level in the CNS. Further elucidation of the mechanisms of cannabinoid modulation of both Th1 and Th2 cytokine expression is required to resolve these questions. Both AEA and $\Delta^{9}$-THC, and potentially $\mathrm{R}(+)$ WIN55,212, can also modulate the immune system by regulating the hypothalamo-pituitary axis via CB1 receptors expressed in the hippocampus (35).

The mechanisms underlying the antiproliferative effects of cannabinoids on $\mathrm{T}$ cell responses both in vitro and in vivo are still unclear, but appear to be dependent upon timing, dose, and the agonist used $(36,37)$. Here we report that $\mathrm{R}(+)$ WIN55,212 treatment at the time of infection can inhibit the proliferation of $\mathrm{VP} 2_{70-86}$-specific $\mathrm{T}$ lymphocytes. $\mathrm{R}(+)$ WIN55,212-treatment at the onset of clinical disease or during established disease, however, had little effect on the proliferative capacity of either VP2 ${ }_{70-86^{-}}$or $\mathrm{PLP}_{139-151^{-}}$-specific T cells. This may reflect differences in cannabinoid efficacy, dependent upon the activation state of the target $T$ cell. The mRNA coding for $\mathrm{CB}$ receptors on $\mathrm{T}$ cells has been shown to be either increased or decreased depending on the activating stimuli, and this may account for some of the differences seen between the early and late treatments (38). Therefore, it appears that activated $\mathrm{T}$ cells may be more refractory to the inhibitory effect of $\mathrm{R}(+)$ WIN55,212 than naive $\mathrm{T}$ cells. This may also explain the lack of inhibitory effect of R(+)WIN55,212 on IFN- $\gamma$ secretion during the late stage of TMEV-IDD. To determine whether $\mathrm{R}(+)$ WIN55,212 could also inhibit clinical disease by blockade of Th1 homing to the CNS, we characterized CNS inflammatory infiltrates. Despite the inhibition of Th1 differentiation, immunohistochemical analysis of the CNS following $\mathrm{R}(+)$ WIN55,212 treatment suggested that $\mathrm{CD}^{+}$, $\mathrm{CD}^{+}, \mathrm{F} 4 / 80$, and $\mathrm{B} 220^{+}$cells were present in numbers equivalent to that of untreated or S(-)WIN55,212treated mice, despite no evidence of increasing severity of clinical disease (Figure 1) and decreased levels of mRNA encoding proinflammatory cytokines in the CNS (Figure 6). At the time of disease onset or during late-stage disease, the CNS will already contain inflammatory infiltrate because disease is ongoing before treatment begins. Therefore, R(+)WIN55,212 may be inhibiting (a) the further polarization of virus and/or myelin epitope-specific Th1 cells in the peripheral lymphoid organs and/or (b) the Th1 cytokine responses in the target organ, i.e., the CNS, either directly at the $T$ cell level or by affecting infiltrating or resident CNS APCs, or both. This observation indicates that the primary effect of the therapy in ongoing disease is at the effector stages of the inflammatory cascade. Collectively, $\mathrm{R}(+)$ WIN55,212 influences clinical disease by inhibiting peripheral Th1 differentiation, but not by preventing proliferative or homing capabilities of activated Th1 cells.

Viral infection is thought to play a role in both the initiation and exacerbation/relapse of MS $(10,11)$. Our results show that $\mathrm{R}(+)$ WIN55,212 inhibits the immune response to the immunodominant TMEV peptide, VP2 ${ }_{70-86}$; therefore, we determined the effect of R(+)WIN55,212 on the susceptibility of mice to TMEV infection. Following early $\mathrm{R}(+)$ WIN55,212 treatment (day 0 ), there was a marked increase in CNS TMEV titers that may be explained by the downregulation of antiviral Th1 responses (Figures 4 and 5). In addition, CNS levels of spinal cord mRNA coding for the antiviral molecules, IFN- $\alpha$ and IFN- $\beta$, were significantly decreased in $\mathrm{R}(+)$ WIN55,212-treated mice (Figure $6 a$ ). This is similar to the suppression of IFN- $\alpha / \beta$ seen in $\Delta^{9}$-THC-treated mice infected with herpes simplex virus (39). Interestingly, despite the increased CNS viral load in $\mathrm{R}(+)$ WIN55,212-treated mice, there was decreased clinical disease, confirming our previous data showing the immune response to TMEV initiates CNS damage, not the cytotoxic capacity of the virus itself $(15,16)$. Infectious CNS TMEV titers reach a 
maximum 10-12 days after infection and then diminish to a persistent low level by day 30 after infection (40), which may explain the lack of difference in viral titers between the $\mathrm{R}(+)$ WIN55,212 and controls in mice following treatment at the onset of clinical disease (postinfection day 26-31). Therefore, viral titers at later time points are unaffected by the R(+)WIN55,212induced inhibition of antiviral immunity.

Previous studies have investigated the immunosuppressive potential of cannabinoids in animal disease models. Dexanabinol (HU-211), $\Delta^{8}$ - and $\Delta^{9}$-THC have been shown to be effective at inhibiting EAE (41-43). Although histological examination showed reduced CNS inflammation, no immunological parameters or mechanisms of action were elucidated. Cannabinoids have also been shown to inhibit tremor and spasticity symptoms in EAE $(8,9)$. In addition, oral administration of cannabidiol, a nonpsychoactive constituent of cannabis, inhibited murine collagen-induced arthritis by inhibiting $\mathrm{T}$ cell proliferation and Th 1 (IFN- $\gamma$ and TNF- $\alpha$ ) cytokine secretion (44).

Further studies are underway to fully determine the mechanisms of therapeutic action of R(+)WIN55,212 in the TMEV model. In addition to the inhibitory effects on the immune system, the therapeutic effect of $\mathrm{R}(+)$ WIN55,212 at the late time point (postinfection day 50-55) may also involve other protective mechanisms. During late-stage EAE, caspase-3 is upregulated and is thought to play an important role in the apoptosis of axons, which leads to the severe hindlimb paralysis seen in that model (45). Caspase-3 is also implicated in the apoptosis of oligodendrocytes (46). It has been shown recently that cannabinoids, including $\mathrm{R}(+)$ WIN55,212, can inhibit caspase-3 activity and may protect mice from severe clinical disability (45). In addition to the anti-inflammatory and neuroprotective properties of cannabinoids, they have also recently been shown to promote oligodendrocyte survival (47) and inhibit glutamate release (48) in the CNS, which has been implicated in neurotoxicity (49). Therefore, although the $\mathrm{T}$ cell component of the TMEV-induced disease may be refractory to the effects of $\mathrm{R}(+)$ WIN55,212 at the late time point, cannabinoid therapy may well affect other ongoing disease processes induced during the chronic stages of TMEV-IDD. We are currently undertaking studies in the TMEV model to determine whether caspase-3 plays a similar role in pathology as was shown in the EAE model.

The use of specific CB receptor antagonists may help elucidate whether the therapeutic effect seen is CB1 and/or CB2 receptor mediated. The current CB receptor antagonists, however, are thought to be "inverse agonists" and capable of delivering a "biological signal" $(50,51)$; thus, it may be difficult to interpret results seen with these reagents. Furthermore, it is currently unknown what effect TMEV-IDD has on the expression of the cannabinoid receptors on the numerous cell types that express them. In EAE, mRNA coding for $\mathrm{CB} 1$ receptors is reduced in certain areas of the brain during disease, although the efficiency of WIN55,212 binding to CB1 is increased (52). Therefore, the effects of exogenous cannabinoids administered during late disease will likely induce different effects because signaling may be occurring only in specific areas of the brain. Furthermore, the expression of CB receptors in the spinal cord during either EAE or TMEV disease is unknown. The spinal cord is the major target for demyelination, and most lesions are located in the white matter. Therefore, it is possible that cannabinoid signaling will be different between the early, onset, and late phases of disease. Finally, studies have determined that endocannabinoid release is upregulated in the brain, and more significantly in the spinal cord, during chronic spastic EAE (8). Therefore, this may also play a role in inducing differential exogenous cannabinoid signaling in the TMEV-IDD model. The presence of additional CB-like receptors may further confuse the issue (53). Therefore, the use of CBreceptor knockout mice and $\mathrm{CB}$ receptor-specific ligands should provide important clues as to further mechanisms and the cell types involved.

A recent, small, randomized, double-blind study on the efficacy and safety of orally administered cannabinoids in MS patients found that Marinol ( $\Delta^{9}$-THC) was safe, although a number of mild side effects were reported (22). Little relief of spasticity after treatment was reported, however. This may be due to the oral route of administration employed since it has been shown that the bioavailability of $\Delta^{9}$-THC is significantly higher following intravenous administration due to "first pass" effects of the gut and liver following oral ingestion (54). Our study and others suggest that other routes of administration, such as intravenous or intraperitoneal, may increase the bioavailability and therefore the efficacy of cannabinoids in treatment regimens $(8,9)$. Indeed, a recent phase III clinical trial has shown that administration of whole-plant medicinal cannabis extract containing THC and cannabidiol as its principal components into the lungs of MS patients by an inhaler significantly increased the efficacy in the symptomatic treatment of spasticity and neuropathic pain (http://www.gwpharm.com/). Correlation of serum levels of $\mathrm{R}(+)$ WIN55,212 between mice and humans is difficult. R(+)WIN55,212 has not been tested in humans, and correlation to existing data for the levels of $\Delta^{9}$-THC in humans is confounded by the fact that drug concentrations in biological fluids are affected by the size of the dose, route of administration, the long-term pattern of drug use, and the individuals' metabolism and excretion rate. In addition, correlation is difficult due to the different structural properties of $\Delta^{9}$-THC and R(+)WIN55,212, and the affinity and therefore the efficacy of $\Delta^{9}$-THC and $\mathrm{R}(+)$ WIN55,212 for either CB1 or CB2 receptors. Furthermore, other compounds in cannabis may synergize with $\Delta^{9}$-THC in vivo, or may actually be metabolized to $\Delta^{9}$-THC (55). Therefore, the quantity of THC present in cannabis extracts used for human clinical 
trials may not give an accurate indication of the actual bioactive quantity of $\Delta^{9}$-THC. Finally, the metabolic rate of the mouse is higher than that of humans. Therefore, a direct comparison of serum levels again may not give an accurate correlation. One way to indirectly compare $\Delta^{9}$-THC levels in humans to $\mathrm{R}(+)$ WIN55,212 in mice would be to determine the quantity of compound needed to induce psychoactive effects in the subjects. In this study we observed psychoactive effects with $20 \mathrm{mg} / \mathrm{kg} \mathrm{R}(+)$ WIN55,212, but not with 5 or $1 \mathrm{mg} / \mathrm{kg}$. In correlation, reported side effects of cannabinoid treatment in humans (60 $\mathrm{mg} / \mathrm{kg} /$ day orally of nabilone, synthetic $\Delta^{9}$-THC) include dizziness and euphoria, both psychoactive effects, therefore indirectly implying that $20 \mathrm{mg} / \mathrm{kg}$ $\mathrm{R}(+)$ WIN55,212 in mice is within the range used for human clinical trials (56).

In summary, treatment with the cannabinoid $\mathrm{R}(+)$ WIN55,212 effectively ameliorates progression of established TMEV-IDD, in part by inhibiting the Th1 differentiation as measured by suppressed antigen-specific DTH, IFN- $\gamma$ secretion, and by inhibiting the production of the proinflammatory cytokines TNF- $\alpha$, IFN- $\gamma$, IL-1 $\beta$, and IL- 6 , in the CNS, which are necessary for the induction and progression of clinical disease (summarized in Table 1). In addition, R(+)WIN55,212 may inhibit clinical disease at multiple levels depending upon the stage of disease. During late-stage disease, $\mathrm{R}(+)$ WIN55,212 has no inhibitory effect upon $\mathrm{T}$ cell proliferation but may limit effector damage of axons in the CNS. This study provides preclinical evidence suggesting that cannabinoids may be promising therapeutic agents for treating autoimmune disorders such as MS by exerting potent immunoregulatory effects, in addition to providing symptomatic relief of spasticity, neuropathic pain, and bladder dysfunction.

\section{Acknowledgments}

This work was supported in part by United States Public Health Service grant NS-23349. J.L. Croxford is a fellow of the National Multiple Sclerosis Society (postdoctoral research fellowship award FG-1456-A-1).

1. Ota, K., et al. 1990. T-cell recognition of an immunodominant myelin basic protein epitope in multiple sclerosis. Nature. 346:183-187.

2. Goodin, D.S., et al. 2002. Disease modifying therapies in multiple sclerosis - Report of the Therapeutics and Technology Assessment Subcommittee of the American Academy of Neurology and the MS Council for Clinical Practice Guidelines. Neurology. 58:169-178.

3. Devane, W.A., Dysarz, F.A., III, Johnson, M.R., Melvin, L.S., and Howlett, A.C. 1988. Determination and characterization of a cannabinoid receptor in rat brain. Mol. Pharmacol. 34:605-613.

4. Munro, S., Thomas, K.L., and Abushaar, M. 1993. Molecular characterization of a peripheral receptor for cannabinoids. Nature. 365:61-65.

5. Galiegue, S., et al. 1995. Expression of central and peripheral cannabinoid receptors in human immune tissues and leukocyte subpopulations. Eur. J. Biochem. 232:54-61.

6. Klein, T.W., Newton, C., and Friedman, H. 1998. Cannabinoid receptors and immunity. Immunol. Today. 19:373-381.

7. Croxford, J.L. 2003. Therapeutic potential of cannabinoids in CNS disease. CNS Drugs. 17:179-202.

8. Baker, D., et al. 2001. Endocannabinoids control spasticity in a multiple sclerosis model. FASEB J. 15:300-302.

9. Baker, D., et al. 2000. Cannabinoids control spasticity and tremor in a multiple sclerosis model. Nature. 404:84-87.
10. Olson, J.K., Croxford, J.L., and Miller, S.D. 2001. Virus-induced autoimmunity: potential role of viruses in initiation, perpetuation, and progression of $\mathrm{T}$ cell-mediated autoimmune diseases. Viral Immunol. 14:227-250.

11. Kurtzke, J.F. 1993. Epidemiologic evidence for multiple sclerosis as an infection. Clin. Microbiol. Rev. 6:382-427.

12. Pevear, D.C., Calenoff, M., Rozhon, E., and Lipton, H.L. 1987. Analysis of the complete nucleotide sequence of the picornavirus Theiler's murine encephalomyelitis virus indicates that it is closely related to cardioviruses. J. Virol. 61:1507-1516.

13. Lipton, H.L. 1975. Theiler's virus infection in mice: an unusual biphasic disease process leading to demyelination. Infect. Immun. 11:1147-1155.

14. Miller, S.D., and Gerety, S.J. 1990. Immunologic aspects of Theiler's murine encephalomyelitis virus (TMEV)-induced demyelinating disease. Semin. Virol. 1:263-272.

15. Karpus, W.J., Pope, J.G., Peterson, J.D., Dal Canto, M.C., and Miller, S.D. 1995. Inhibition of Theiler's virus-mediated demyelination by peripheral immune tolerance induction. J. Immunol. 155:947-957.

16. Miller, S.D., et al. 1997. Persistent infection with Theiler's virus leads to CNS autoimmunity via epitope spreading. Nat. Med. 3:1133-1136.

17. Croxford, J.L., et al. 1998. Cytokine gene therapy in experimental allergic encephalomyelitis by injection of plasmid DNA-cationic liposome complex into the central nervous system. J. Immunol. 160:5181-5187.

18. Croxford, J.L., et al. 2000. Gene therapy for chronic relapsing experimental allergic encephalomyelitis using cells expressing a novel soluble p75 dimeric TNF receptor. J. Immunol. 164:2776-2781.

19. Croxford, J.L., Feldmann, M., Chernajovsky, Y., and Baker, D. 2001. Different therapeutic outcomes in experimental allergic encephalomyelitis dependent upon the mode of delivery of IL-10: a comparison of the effects of protein, adenoviral or retroviral IL-10 delivery into the central nervous system. J. Immunol. 166:4124-4130.

20. Olson, J.K., Eagar, T.N., and Miller, S.D. 2002. Functional activation of myelin-specific T cells by virus-induced molecular mimicry. J. Immunol. 169:2719-2726.

21. Begolka, W.S., Vanderlugt, C.L., Rahbe, S.M., and Miller, S.D. 1998. Differential expression of inflammatory cytokines parallels progression of central nervous system pathology in two clinically distinct models of multiple sclerosis. J. Immunol. 161:4437-4446.

22. Killestein, J., et al. 2002. Safety, tolerability, and efficacy of orally administered cannabinoids in MS. Neurology. 58:1404-1407.

23. Jacobs, C.A., et al. 1991. Experimental autoimmune encephalomyelitis is exacerbated by IL-1 alpha and suppressed by soluble IL-1 receptor. J. Immunol. 146:2983-2989.

24. Samoilova, E.B., Horton, J.L., Hilliard, B., Liu, T.S., and Chen, Y. 1998. IL-6-deficient mice are resistant to experimental autoimmune encephalomyelitis: roles of IL- 6 in the activation and differentiation of autoreactive T cells. J. Immunol. 161:6480-6486.

25. Selmaj, K., Raine, C.S., and Cross, A.H. 1991. Anti-tumor necrosis factor therapy abrogates autoimmune demyelination. Ann. Neurol. 30:694-700.

26. Qi, Y., and Dal, C.M. 1996. Effect of Theiler's murine encephalomyelitis virus and cytokines on cultured oligodendrocytes and astrocytes. J. Neurosci. Res. 45:364-374.

27. Matias, I., et al. 2002. Presence and regulation of the endocannabinoid system in human dendritic cells. Eur. J. Biochem. 269:3771-3778.

28. Yuan, M., et al. 2002. Delta 9-tetrahydrocannabinol regulates Th1/Th2 cytokine balance in activated human $\mathrm{T}$ cells. J. Neuroimmunol. 133:124-131.

29. Molina-Holgado, F., Molina-Holgado, E., and Guaza, C. 1998. The endogenous cannabinoid anandamide potentiates interleukin- 6 production by astrocytes infected with Theiler's murine encephalomyelitis virus by a receptor-mediated pathway. FEBS Lett. 433:139-142.

30. Molina-Holgado, F., Lledo, A., and Guaza, C. 1997. Anandamide suppresses nitric oxide and TNF-alpha responses to Theiler's virus or endotoxin in astrocytes. Neuroreport. 8:1929-1933.

31. Sanjabi, S., Hoffmann, A., Liou, H.C., Baltimore, D., and Smale, S.T. 2000. Selective requirement for c-Rel during IL-12 P40 gene induction in macrophages. Proc. Natl. Acad. Sci. U. S. A. 97:12705-12710.

32. Newton, C.A., Klein, T.W., and Friedman, H. 1994. Secondary immunity to Legionella pneumophila and Th1 activity are suppressed by delta9-tetrahydrocannabinol injection. Infect. Immun. 62:4015-4020.

33. Rowell, J.F., and Griffin, D.E. 1999. The inflammatory response to nonfatal Sindbis virus infection of the nervous system is more severe in SJL than in BALB/c mice and is associated with low levels of IL- 4 mRNA and high levels of IL-10-producing CD4+ T cells. J. Immunol. 162:1624-1632.

34. Strle, K., et al. 2001. Interleukin-10 in the brain. Crit Rev. Immunol. 21:427-449.

35. Weidenfeld, J., Feldman, S., and Mechoulam, R. 1994. Effect of the brain constituent anandamide, a cannabinoid receptor agonist, on the hypothalamo-pituitary-adrenal axis in the rat. Neuroendocrinology. 59:110-112.

36. Klein, T.W., Friedman, H., and Specter, S. 1998. Marijuana, immunity and infection. J. Neuroimmunol. 83:102-115. 
37. Parolaro, D. 1999. Presence and functional regulation of cannabinoid receptors in immune cells. Life Sci. 65:637-644.

38. Noe, S.N., Newton, C., Widen, R., Friedman, H., and Klein, T.W. 2000 Anti-CD40, anti-CD3, and IL-2 stimulation induce contrasting changes in CB1 mRNA expression in mouse splenocytes. J. Neuroimmunol. 110:161-167.

39. Cabral, G.A., Lockmuller, J.C., and Mishkin, E.M. 1986. Delta 9-tetrahydrocannabinol decreases alpha/beta interferon response to herpes simplex virus type 2 in the B6C3F1 mouse. Proc. Soc. Exp. Biol. Med. 181:305-311.

40. Trottier, M., Kallio, P., Wang, W., and Lipton, H.L. 2001. High numbers of viral RNA copies in the central nervous system of mice during persistent infection with Theiler's virus. J. Virol. 75:7420-7428.

41. Wirguin, I., et al. 1994. Suppression of experimental autoimmune encephalomyelitis by cannabinoids. Immunopharmacology. 28:209-214.

42. Lyman, W.D., Sonett, J.R., Brosnan, C.F., Elkin, R., and Bornstein, M.B. 1989. Delta 9-tetrahydrocannabinol: a novel treatment for experimental autoimmune encephalomyelitis. J. Neuroimmunol. 23:73-81.

43. Achiron, A., Miron, S., Lavie, V., Margalit, R., and Biegon, A. 2000. Dexanabinol (HU-211) effect on experimental autoimmune encephalomyelitis: implications for the treatment of acute relapses of multiple sclerosis. J. Neuroimmunol. 102:26-31.

44. Malfait, A.M., et al. 2000. The nonpsychoactive cannabis constituent cannabidiol is an oral anti-arthritic therapeutic in murine collageninduced arthritis. Proc. Natl. Acad. Sci. U. S. A. 97:9561-9566.

45. Ahmed, Z., et al. 2002. A role for caspase- 1 and -3 in the pathology of experimental allergic encephalomyelitis: inflammation versus degeneration. Am. J. Pathol. 161:1577-1586.

46. Hisahara, S., Yuan, J., Momoi, T., Okano, H., and Miura, M. 2001. Caspase-11 mediates oligodendrocyte cell death and pathogenesis of autoimmune-mediated demyelination. J. Exp. Med. 193:111-122.

47. Molina-Holgado, E., et al. 2002. Cannabinoids promote oligodendrocyte progenitor survival: involvement of cannabinoid receptors and phosphatidylinositol-3 kinase/Akt signaling. J. Neurosci. 22:9742-9753.

48. Hampson, A.J., Grimaldi, M., Axelrod, J., and Wink, D. 1998. Cannabidiol and (-)Delta9-tetrahydrocannabinol are neuroprotective antioxidants. Proc. Natl. Acad. Sci. U. S. A. 95:8268-8273.

49. Werner, P., Pitt, D., and Raine, C.S. 2000. Glutamate excitotoxicity-a mechanism for axonal damage and oligodendrocyte death in multiple sclerosis? J. Neural Transm. Suppl. 60:375-385.

50. MacLennan, S.J., Reynen, P.H., Kwan, J., and Bonhaus, D.W. 1998. Evidence for inverse agonism of SR141716A at human recombinant cannabinoid CB1 and CB2 receptors. Br. J. Pharmacol. 124:619-622.

51. Bouaboula, M., et al. 1997. A selective inverse agonist for central cannabinoid receptor inhibits mitogen-activated protein kinase activation stimulated by insulin or insulin-like growth factor 1 . Evidence for a new model of receptor/ligand interactions. J. Biol. Chem. 272:22330-22339.

52. Berrendero, F., et al. 2001. Changes in cannabinoid $\mathrm{CB}(1)$ receptors in striatal and cortical regions of rats with experimental allergic encephalomyelitis, an animal model of multiple sclerosis. Synapse. 41:195-202.

53. Brooks, J.W., et al. 2002. Arvanil-induced inhibition of spasticity and persistent pain: evidence for therapeutic sites of action different from the vanilloid VR1 receptor and cannabinoid $\mathrm{CB}(1) / \mathrm{CB}(2)$ receptors. Eur. J. Pharmacol. 439:83-92.

54. Agurell, S., et al. 1986. Pharmacokinetics and metabolism of delta 1-tetrahydrocannabinol and other cannabinoids with emphasis on man. Pharmacol. Rev. 38:21-43.

55. McPartland, J.M., and Pruitt, P.L. 1999. Side effects of pharmaceuticals not elicited by comparable herbal medicines: the case of tetrahydrocannabinol and marijuana. Altern. Ther. Health Med. 5:57-62.

56. Chan, H.S., Correia, J.A., and MacLeod, S.M. 1987. Nabilone versus prochlorperazine for control of cancer chemotherapy-induced emesis in children: a double-blind, crossover trial. Pediatrics. 79:946-952. 